\title{
Development and Pilot Implementation of a Ladderized Biosafety Training Program in a Specialty Infectious Disease Hospital and Research Institute
}

\author{
Plebeian Medina, Abigail Padua, Michelle Casagan, Randy Olpindo, Amado Tandoc III, Socorro Lupisan
}

Research Institute for Tropical Medicine - Department of Health, Philippines

\section{ABSTRACT}

Biosafety is the application of laboratory practices, use of safety equipment and implementation of procedures in laboratory facilities when working with potentially infectious microorganisms to protect not only the laboratory worker, but also the general public and the environment. Biosafety training specifically structured based on risk is vital to establish a safe working environment to reduce the risks of unintentional exposure and/ or intentional release of infectious microorganisms. In 2016, a ladderized 3-step biosafety training program was established by the Research Institute for Tropical Medicine, a specialty infectious disease hospital and National Reference Laboratory in the Philippines. The training program includes 1) Biosafety 101, offered to all new RITM employees; 2) Applied Biosafety training, especially designed for laboratory personnel; and 3) Advanced Biosafety training, focused on developing Biosafety Officers and infectious disease outbreak responders. A 30\% increase in awareness on biosafety has been achieved among participants of the first two steps of the program, with the third module to be implemented in 2017.

Key words : biosafety, biosafety training program, biosecurity

ISSN (0118-3265

Printed in the Philippines.

Copyright $\odot 2017$ by the PJP.

Received: 17 October 2016.

Accepted: 7 December 2016.

Published online first: January 21, 2017.

https://doi.org/10.21141/PJP.2017.002

Corresponding author: Abigail A. Padua

E-mail: abiapadua@gmail.com

\section{INTRODUCTION}

In the past, laboratory practices are directly mentored by senior staff as an orientation procedure prior the conduct of laboratory work. To be able to work independently, individuals must demonstrate successful and acceptable behavior within the laboratory according to the evaluation and trust of senior and experienced laboratory personnel.

Emergence of antimicrobial resistance and highly infectious viral pathogens including MERS CoV, Ebola and Zika virus, comprise health threats that challenge both the public health sector and biosafety experts worldwide. Collection, handling, testing, transportation, storage and disposal of clinical specimens from cases suffering from emerging infectious diseases present significant risks to health care personnel, the community and the environment.

Biosafety is the discipline that addresses the safe handling and containment of infectious microorganisms and hazardous biological materials. ${ }^{1}$ The goal of biosafety is to minimize the risk of infection to individual laboratory workers and reduce the accidental or unintentional release of pathogens from the laboratory to the community. ${ }^{2}$ It is influenced by the impact of emerging and reemerging infectious diseases while serving the interest of global public health and protecting laboratory workers all at the same time. Ensuring safe working conditions in laboratories should be based on identified risks, among which are the lack of biosafety and biosecurity awareness and weak implementation of standard biosafety practices. ${ }^{3}$ As such, there is a necessity to develop and implement a comprehensive biosafety training program that not only accounts for the risks posed by pathogens and pathogenic materials handled by the laboratory, and its existing facilities and biosafety controls, but also the capability of personnel in identifying and mitigating these risks. 


\section{Risk classification for laboratories}

Laboratory manipulation of microorganisms can be safely conducted in either a basic laboratory, containment facility, or maximum containment facility based on risk assessment. The World Health Organization (WHO) and US National Institutes of Health (NIH) has recommended pathogens classification for laboratory use that describes the four general risk groups (1-4) based on its individual characteristics and the route of transmission of the natural disease in relation to biosafety levels (Table 1). Laboratory facilities are divided into four categories. Biosafety Level 1 and Biosafety Level 2 (basic), Biosafety Level 3 (containment), and Biosafety Level 4 (maximum containment). Biosafety level designations are based on a combination of design features, construction, containment facilities, equipment, practices and operational procedures required for working with agents from the various risk groups. ${ }^{1,4}$ The biosafety level requirement for a specific laboratory task shall be based on risk assessment and professional judgment rather than by risk group classification. Work with novel and dangerous pathogens has to be conducted in an appropriate containment facility by trained and competent laboratory personnel.

\section{Biosafety and laboratory biorisk management}

Laboratory biorisk management is the analysis of risks and development of strategies to minimize the likelihood of the occurrence of biorisks, with the overall aim of reducing the risk of accidental exposure to or release of biological hazards. ${ }^{5}$ Biosafety training plays a vital role in this risk management strategy. It provides an effective approach to increase biosafety and biosecurity capacity of laboratory workers in a containment laboratory. ${ }^{2} \mathrm{~A}$ welldefined and structured biosafety training enables the application of these concepts and skills in a formal and systematic manner against emerging highly infectious agents. ${ }^{6}$ Biosafety professionals working in individual institutions are encouraged to develop and implement site specific and standardized biosafety training programs appropriate for their own laboratory facilities.

The CEN Workshop Agreement (CWA 15793: 2011) on Biorisk Management, WHO Biosafety Guidelines, US BMBL and the Canadian Biosafety Standard and Guidelines highlight the importance of biosafety training requirement to ensure biological safety and security.

\section{BACKGROUND OF THE INSTITUTE}

The Research Institute for Tropical Medicine (RITM) was established in 1981 thru Executive Order No. 674 to undertake research activities in the diagnosis, control and prevention of tropical diseases that are major causes of mortality and morbidity in the Philippines; conduct clinical trials aimed at better understanding and control of tropical diseases; conduct regular training courses for medical and paramedical personnel in the control of common tropical diseases in the country; and provide high quality tertiary care to both in-patients and out-patients suffering from tropical diseases included within the scope of the Institute's research activities.

RITM was constructed and equipped by the Japanese government through a Grant-in-Aid of the Japan International Cooperation Agency (JICA) in a 31,000 square meters lot located inside the Filinvest Corporate City, Alabang, Muntinlupa City. Further grant assistance was extended by JICA to RITM for the construction of the Animal Research Laboratories in 1985, Training Center and Residence Hall in 1989, and eventually the National TB Reference Laboratory Building in 2002.

In 1999, production of biologicals and vaccines was added to the mandate of RITM through Executive Order 102, in which the Department of Health Biologicals Production Service (BPS) was merged with the institute.

In November 2000, the DOH, thru the issuance of Department Order No. 393-E, in addition to the functions stated above, designated the Research Institute for Tropical Medicine as the National Reference Laboratory (NRL) for dengue and other arboviruses, influenza and other respiratory viruses, tuberculosis and other Mycobacteria, malaria and other parasites, bacterial enteric diseases, measles and other viral exanthems, mycology, polio and other enteroviruses, antimicrobial resistance, emerging bacterial diseases, and confirmatory testing of blood units. RITM is also the designated National Referral Center for Management of Severe Acute Respiratory Syndrome (SARS) and Other Emerging and Re-emerging Infectious Diseases (DOH-Department Order No. 698 s. 2004). In 2014, RITM was officially designated as the Philippine National Influenza Center (PNIC). The following year, RITM was officially designated as National Reference Laboratory for Rotavirus and other Enteric viruses. Through the DOH Committee on NRLs of the National Health Laboratory Network Initiative, RITM shall be further designated as NRL for Schistosomiasis, Neglected Tropical Parasitic Diseases, Rabies and other Lyssaviruses, Special Pathogens, Invasive Bacterial Vaccine Preventable Diseases, and Public Health Entomology. Specialized laboratories were also established to further improve the service of the institute in response to emerging and re-emerging infectious pathogens, like the National Tuberculosis Reference Laboratory (NTRL), Special Pathogens Laboratory (SPL) and the Molecular Biology Laboratory (MBL).

With these highly-specialized facilities, it is imperative that the institute lay down and implement stringent biosafety and biosecurity policies.

\section{RITM BIOSAFETY TRAINING PROGRAM}

Biosafety training has previously been dependent on each laboratory department's separate orientation and training programs. With no standard training material, there is a potential for inconsistencies

Table 1. WHO and NIH risk group classification in relation to biosafety levels, practices and equipment

\begin{tabular}{|c|c|c|c|c|}
\hline Risk Group & Biosafety Level & Laboratory Type & Laboratory Practices & Safety Equipment \\
\hline 1 & Basic-Biosafety Level 1 & Basic teaching, research & $\begin{array}{l}\text { Good microbiological techniques } \\
\text { (GMT) }\end{array}$ & None; open bench work \\
\hline 2 & Basic-Biosafety Level & $\begin{array}{l}\text { Primary Health Services, } \\
\text { diagnostic services, research }\end{array}$ & $\begin{array}{l}\text { GMT plus protective clothing, } \\
\text { biohazard sign }\end{array}$ & $\begin{array}{l}\text { Open bench plus biological safety cabinet } \\
\text { (BSC) for potential aerosols }\end{array}$ \\
\hline 3 & $\begin{array}{l}\text { Containment-Biosafety } \\
\text { Level } 3\end{array}$ & $\begin{array}{l}\text { Special diagnostic services, } \\
\text { research }\end{array}$ & $\begin{array}{l}\text { Level } 2 \text { plus special clothing, } \\
\text { controlled access, directional airflow }\end{array}$ & $\begin{array}{l}\text { BSC and/or other primary devices for all } \\
\text { activities }\end{array}$ \\
\hline 4 & $\begin{array}{l}\text { Maximum containment- } \\
\text { Biosafety Level } 4\end{array}$ & Dangerous pathogen units & $\begin{array}{l}\text { Level } 3 \text { plus air lock entry, exit } \\
\text { showers, special waste disposal }\end{array}$ & $\begin{array}{l}\text { Class III BSC or positive pressure suits with } \\
\text { Class II BSCs, double ended autoclaved } \\
\text { (through the wall), filtered air }\end{array}$ \\
\hline
\end{tabular}


on the level of awareness and competence. As the number of individuals working with infectious microorganism increases, standardized introductory biosafety training may be helpful, thus, a sustainable, centralized, and reproducible system needs to be developed and improved. A ladderized training program has been set to create different levels of training and awareness among RITM employees depending on their specific role within the institute.

An institutional biosafety committee previously existed under the Assistant Director's Office which served to establish biosafety and biosecurity policies. However, the committee suffered attrition in recent years due to retirement of key staff. In 2016, the Director's Office established the Biorisk Management Office (BRMO). The BRMO, with the vision of making RITM the leader of applied biosafety and biosecurity in the Philippines, is tasked to implement policies, monitor their implementation, conduct risk assessments, and evaluate biosafety and biosecurity programs within the institute.

The BRMO developed a new institutional ladderized biosafety training course, designed as a 3-ladder step program which includes the following: an introductory course offered to all new employees, regardless of whether they are engaged in any laboratory activities or not. In-house guidelines have also been developed to familiarize workers on biosafety and biosecurity; the Applied biosafety training program, the second step of the program, is designed for in house laboratory personnel tasked to conduct routine and special laboratory procedures, such as, collection, handling, testing, storage and disposal of specimens, isolates and biologicals; the third part of the program is the Advanced Biosafety training, which focuses on developing biosafety officers and infectious disease outbreak responders. A regular monthly meeting of biosafety officers is conducted to provide a proper platform for the sharing of newly acquired skills and knowledge, as well as discussion of relevant concerns.

As reference for the development of the ladderized training program, the office incorporated the following critical elements for an effective biosafety training program according to the WHO laboratory biosafety manual of 2004:

- Needs assessment: Determination of tasks to be carried out as well as the proper approach for each task.

- Establishment of training objectives: Identification of observable behaviors, capabilities and level of proficiency that are expected to be demonstrated by the trainees at the end of the program.

- Specification of training content and media: Design of media to be used in conducting training. It must be comprehensive and efficient to be able to provide the intended knowledge and skills to the trainees.

- Accounting for individual learning differences: Incorporation of different training approaches in developing a biosafety training program is vital since each individual has different learning capabilities.

- Specifying learning conditions: Selection of a mode of instruction is important in passing the required information to the trainees. The different approaches to be used should deliver the expected outcomes of the training.

- Training evaluation: This should determine the effectivity of the training as measured by the achievement of the overall goal.

- Training revision: The use of questionnaires and survey methods to evaluate the overall training process are necessary to improve future training programs.

\section{DEVELOPMENT OF THE PROGRAM}

The development of the Biosafety training aims to adapt a centralized biosafety and biosecurity orientation that will be applicable to all RITM personnel. The training shall be conducted by both traditional lecture and non-traditional training methods customized to the adult learning process and strategies. The flow chart on Figure 1 shows the detailed step by step process on the creation of this ladderized biosafety training program.

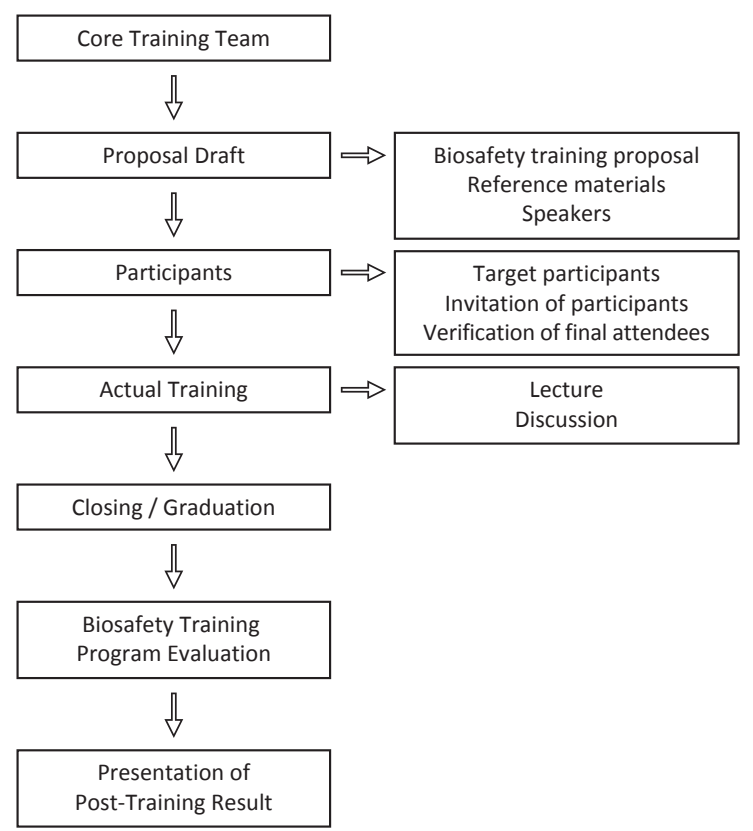

Figure 1. Process flow for the development of the Ladderized Biosafety Training Program.

First, the creation of the core training team which is composed of consultants and Biosafety officers. The team is responsible for the planning, organization and to facilitate the execution of the whole program. A draft proposal was then conceptualized with the use of RITM biosafety manuals with the aid of the previously mentioned international biosafety manuals as reference materials. After which the target speakers which are all biosafety officers, will then be tapped for the program. Guidelines for determination of participants were also drafted. Each Biosafety training level targets different levels of participants. The actual training program will then be carried out and was evaluated based on the evaluation guidelines that were prepared.

\section{Initial Assessment}

Participants took a written examination before the start of the Applied Biosafety training. The test aimed to assess the knowledge of each participant on the concepts on biosafety and biosafety hazards associated with the facility as well as to determine the training needs and approaches to be used. The set of questionnaires is based on the content that will be covered in the entire program.

\section{Level 1: Biosafety 101}

Biosafety 101 is an introductory course open for all employees regardless of its role in a laboratory containment facility. It was established in the premise that as one institution, employees work towards one mission and therefore share one risk. The information on how to mitigate the risk must therefore be understood by all. Biosafety 101 aims, not only to raise awareness on the basic and fundamental principles of biological safety and biosecurity but to direct employees' perception of the risk that this institution shares 
when fulfilling its role in infectious disease outbreak investigation and national health emergencies.

Biosafety 101 is presented and facilitated by trained and experienced biosafety officers and professionals through a comprehensive and informative didactic, lecture and question and answer at the end of each session. The course covers the following topics:

- Fundamentals of Biosafety / Biosecurity, Code of Practice and Best BSL2 Practices

- Biological Risk Management

- Risk Assessment

- Personal Protective Equipment

- Biological Safety Cabinet

- Infectious Substance Shipping and Specimen Transport

- Biological Waste Disposal

- Animal Biosafety

- Arthropod Biosafety

- Infection Control

- Chemical Safety

- Emergency Response

Biosafety 101 as the initial step in the ladder aims to establish the employees' baseline awareness on the basic principles of biological safety and security that could facilitate employees understanding in its role in the mission of the institute of providing safe and secured working environment.

\section{Level 2: Applied Biosafety Training}

Applied biosafety training is dedicated for emplovees who are directly involved in collection, handling, testing, transport, storage and disposal of clinical specimen, isolates and biological materials. It aims to ensure compliance with the standard biosafety practices based on risk assessment. This uses the adult learning teaching strategies to ensure active participation and maximize hands on learning experience. The training is not limited to power point slide presentation, lecture, and question \& answer portion alone. Participants experience applied biosafety through its sessions that are filled with hands on laboratory activities that will stimulate the audience active participation and maximize learning capability. This includes demonstrations and return demonstration of best laboratory practices, video presentations on how safety equipment works, case study scenario, actual risk assessment process, performance evaluation process, group exercises, reporting and open discussion. Applied biosafety training is facilitated by home grown biosafety officers, invited biosafety professionals and experts who are trained in facilitating adult learning and teaching strategies. The Applied Biosafety Training is a three day workshop covering the following topics:

- Review of the basic principles of Biosafety and Biosecurity

- Microbiological Risk Assessment

- Engineering Mitigation Control: Facility, Biosafety Cabinet and other Safety Equipment

- Personal Protective Equipment

- Operational Practices

- BSL-1, 2 and 3 Practices

- Sterilization and Disinfection

- Infectious Substance Shipping based on IATA regulation

- Emergency Response

- Biological Spill Drill and other emergencies related and limited to biosafety

- Biorisk Performance Evaluation

- Biosafety Audit and Inspection

- Establishing and Understanding Biological Safety Culture in an Institution

\section{Level 3: Advanced Biosafety Training}

Advanced biosafety training is of two forms, the Advanced Biosafety Training program for Biosafety Officers and the Advanced Biosafety Training program for laboratory outbreak responders.

The Advanced Biosafety Training for Outbreak Responders is limited for Laboratory Research Division appointed personnel who will be task to be first line, second and third line responders at the event of an emerging disease outbreak. Appointed LRD personnel shall undergo three whole day intensive biorisk management session. The training program ensures risk assessment based approach in selecting the most appropriate control measures at a given situation at the time of outbreak and biological emergency. This will enable the participants to review and apply best biosafety practices to reduce the risk of exposure. This training program aims to strengthen camaraderie and coordination among all biosafety officers nationwide as well as to promote professional biosafety judgment.

Advanced biosafety training for biosafety officers is exclusive for the selected/appointed laboratory personnel of each department who undergone meticulous screening selection process, biosafety train the trainer programs and completed the first two steps of this ladderized program. Biosafety Officers will serve for a minimum of 2 years- on the job-training program upon the release of office order. Biosafety Officers training program is focused on biorisk management system according to CEN Workshop Agreement 15793:2011. Biosafety Officers shall deal with the implementation of Biosafety/Biosecurity administrative controls. This includes institutional biosafety risk assessment and management, hazard communication and biosafety protocol review. In addition, monitoring of biorisk performance through audit and inspection and facilitating biosafety training programs are also included. Each biosafety officers are expected to promote a biosafety program under the Biorisk Management Committee that will strengthen the culture of safety in the institute. Upon completion, successful biosafety officers will be qualified to be listed as Registered Biosafety Professionals. RITM Registered Biosafety Professional will be the official members of the Biorisk Management Committee who will ensure continuous improvement of the biorisk management system of the institute.

The sessions for this training include:

- Updates on emerging threats

- Review of laboratory procedure and work flow

- Biological Risk Assessment

- Biorisk Management Plan

- Engineering Control

- Rational Use and Selection of Personal Protective Equipment - Respiratory Fit Testing

- Use of Biological Safety Cabinets

- Decontamination Procedures

- Response for Biological Spill and other Emergencies

Participants will spend $60 \%$ of the program in a laboratory setting. Responders are expected to master the standard flow of specimen, personnel and wastes, proper donning and doffing procedures, inspection of engineering controls and directional air flow, use of biosafety cabinets and material placement, spill response and procedures in the event of an emergency. Twenty percent $(20 \%)$ of the time will be a workshop on risk assessment and risk management plan. Participants will be provided with different realistic scenarios. The group will develop constructive risk assessment based approach to different biosafety challenges at 
the time of response. The remaining $10 \%$ of the time will be spend for listening and participating to discussion on essential biosafety topics and updates from invited biosafety experts and professionals At the end of the training, appointed responders will be equipped with professional judgment and advanced biosafety skills necessary to reduce the risk and step over the challenges of an emerging biological threat.

\section{Final Assessment}

The final assessment is accomplished through a written examination consisting of the same set of questionnaires that were given during the initial assessment. After the post-test, discussions on the answers were done focusing on the items where the most number of participants performed poorly. Those who fail to take the posttest are required to take a refresher course prior to re-examination. Completion certificates will be issued to successful participants upon completion of each training.

\section{PILOT IMPLEMENTATION}

A total of 118 RITM employees were enrolled for a one-day Biosafety 101 training workshop. All divisions and offices under the institution have cooperated and sent representatives, Janitorial Services, Engineering, Security Support Service, Surveillance Unit, Administrative Office, Clinical Trial Personnel under the Clinical Research Division and the Laboratory Research Division. Majority of the participants are under the laboratory division. This aims to train all employees of RITM, laboratory workers or not. Because of different educational backgrounds, experiences and job description level of familiarity with biosafety guideline varies. Eighty-seven (87) out of 118 trainees composed of the Laboratory Research Division which can be considered more familiar with biosafety guidelines compared to 31 participants from the non-laboratory divisions. A graph representation of the division of the participants is shown on Figure 2.

Applied biosafety training serves as a higher level of biosafety training. The second step of this ladderized program, which prioritizes applied biosafety practices and drills, is especially designed for laboratory workers. It serves a refresher course to maintain previously acquired skills and knowledge as well as to keep updated to new biosafety concepts after the Biosafety 101 training. A total of 40 participants advanced to this training workshop, which is composed of laboratory personnel from the Laboratory Research Division (LRD). Figure representation of the attendees is shown at Figure 3.

As of this publication, the Biorisk Level 3, Advanced Biosafety training is still in process for implementation. Target participants are those who have already completed both Biosafety 101 and Applied Biosafety Training courses. At the end of this 3-step ladder program, it is expected that the successful participants can serve as responders during infectious disease outbreak and related emergencies.

\section{Evaluation of the biosafety training program}

Post-training test scores of all trainees were above the passing mark. A paired t-test was run on the pre- and post-test scores of the participants of the Biosafety training course to determine if there is a statistically significant mean difference between the two sets of exams. As seen in the statistical output generated using the computer tool Stata 13 , there is a statistically significant increase of 30.07 (95\% CI, p<0.05) (Figure 4).

\section{Biosafety 101 Training}

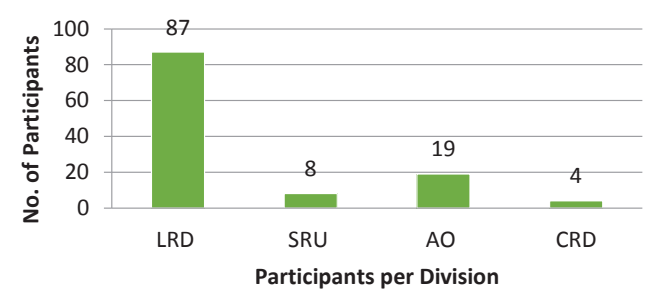

Figure 2. Biosafety 101 training distribution of participants (LRD - Laboratory Research Division; SRU - Surveillance and Response Unit; AO - Administrative Office; CRD - Clinical Research Division).

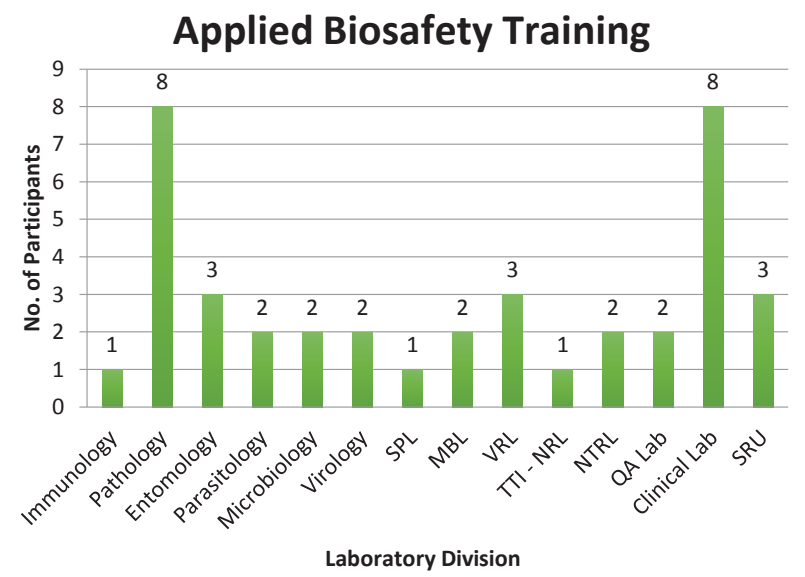

Figure 3. Applied Biosafety training distribution of participants (SPL- Special Pathogens Laboratory; MBL - Molecular Biology Laboratory; VRL - Veterinary Research Laboratory; TTI-NRL - Transfusion Transmitted Infections National Reference Laboratory; NTRL - National Tuberculosis Reference Laboratory; QA - Quality Assurance; SRU - Surveillance and Response Unit).

\section{Applied Biosafety Training}

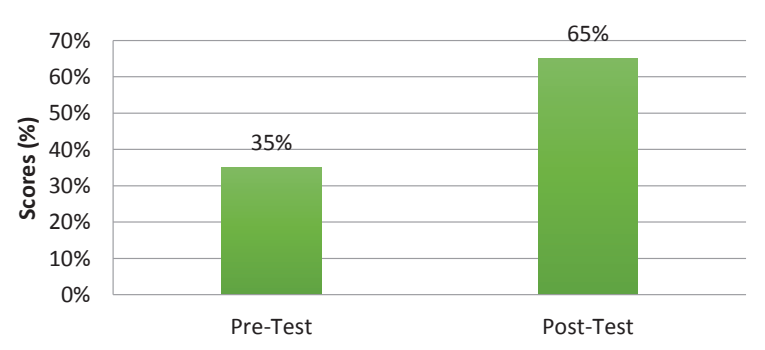

Figure 4. Mean pre-training and post-training scores.

\section{RECOMMENDATIONS}

The authors suggest breaking down the introductory Biosafety 101 course into a series of sessions focusing on 1 to 2 topics per session to improve attendance and allow more in-depth discussion of all the topics, instead of conducting it as a one-day activity. A standard, fit-for-purpose method on the provision of pre- and post-tests for all the 3 levels of the training should be implemented. Demonstration of standard biosafety skills and practices, and its evaluation, should be incorporated under the Applied Biosafety training. Finally, the authors recommend holding an annual refresher course complete with biosafety spill drills, and a module type refresher every two years, to ensure that all participants are up to date. 


\section{ACKNOWLEDGMENTS}

The authors thank the Certified Biosafety Officers of the Philippine Advanced Biorisk Officers Training (PhABOT 3) who facilitated the Biosafety 101 training. They also thank the RITM Department Biosafety Officers for their participation as biosafety trainers for the Applied Biosafety Training. They are grateful to the World Health Organization (WHO) TDR-Supported Regional Training Center and the RITM Laboratory Research Division, Head and staff for their continued support to the program. They extend special thanks to Ms. Rowena Capistrano, head of the Surveillance Unit and to Ms. Johanna Beulah Sornillo, Department of Epidemiology and Biostatistics - RITM for her assistance in this paper's statistical analysis.

\section{AUTHOR DISCLOSURE}

The authors declared no conflicts of interest.

\section{FUNDING SOURCE}

The institute funded the development and implementation of the training program.

\section{REFERENCES}

1. U.S Department of Health and Human Services, Public Health Service, Centers for Disease Control and Prevention, National Institutes of Health. Biosafety in microbiological and biomedical laboratories. 5th ed. Washington, DC: US Dept. of Health and Human Services, Public Health Service, Centers for Disease Control and Prevention, National Institutes of Health, 2009.

2. Inoue S, Bundi M, I Miring'u G, Muriithi B, Ashur S, Wandera E, et al., Evaluation of a BSL-3 laboratory biosafety training program in Kenya. Journal of Biosafety and Biotechnology. 2015;3(4):288-96. Available at: http://jobb.co.in/docs/vol3_ issue4/paper4.pdf.

3. Kaufman SG, Berkelman R. Biosafety "behavioral-based" training for high biocontainment laboratories: bringing theory into practice for biosafety training. Applied Biosafety. 2007; 12 (3): 178-184. Available at: http://journals.sagepub.com/doi/ pdf/10.1177/153567600701200307.

4. World Health Organization. Laboratory biosafety manual. 3rd ed. Geneva: World Health Organization, 2004.

5. World Health Organization. Biorisk management. Laboratory biosecurity guidance: World Health Organization, 2006.

6. Zaki AN, Biosafety and biosecurity measures: management of biosafety level 3 facilities. Int $\mathbf{J}$ of Antimicrob Agents. 2010:36(1):S70-4. https://doi.org/10.1016/j.jjantimicag.2010. 06.026 .

Disclaimer: This journal is OPEN ACCESS, providing immediate access to its content on the principle that making research freely available to the public supports a greater global exchange of knowledge. As a requirement for submission to the PJP, all authors have accomplished an AUTHOR FORM, which declares that the ICMJE criteria for authorship have been met by each author listed, that the article represents original material, has not been published, accepted for publication in other journals, or concurrently submitted to other journals, and that all funding and conflicts of interest have been declared. Consent forms have been secured for the publication of information about patients or cases; otherwise, authors have declared that all means have been exhausted for securing consent. 